\title{
Netrin-1 restores cell injury and impaired angiogenesis in vascular endothelial cells upon high glucose by PI3K/AKT-eNOS
}

\author{
Ying Xing1,*, Jingbo Laî,*, Xiangyang Liu1,*, Nana Zhang1', Jie Ming1, Hengxin Liu² and \\ Xi Zhang ${ }^{2}$
}

1Department of Endocrinology and Metabolism Disease, Xijing Hospital, Forth Military Medical University, Xi'an, Shaanxi, People's Republic of China

Institute of Plastic and Reconstructive Surgery, Xijing Hospital, Forth Military Medical University, Xi'an, Shaanxi, People's Republic of China

*(Y Xing, J Lai and X Liu contributed equally to this work and are defined as the co-first authors)

Correspondence should be addressed to $X$ Zhang

Email

xizhanghdejhd@tom.com

\begin{abstract}
Diabetic foot ulceration (DFU) represents a common vascular complication of diabetes mellitus (DM) with high morbidity and disability resulting from amputation. Netrin-1 level was decreased in type 2 DM patients and has been identified as a protective regulator against diabetes-triggered myocardial infarction and nephropathy. Unfortunately, its role and molecular mechanism in DFU remain poorly elucidated. Here, netrin-1 levels were reduced in DM and DFU patients relative to healthy controls, with netrin-1 levels being the lowest in DFU patients. Moreover, exposure to high glucose (HG) also suppressed netrin-1 expression in human umbilical vein endothelial cells (HUVECs). Elevated netrin-1 expression by infection with Ad-netrin-1 adenovirus vector protected against HUVEC injury in response to HG by ameliorating the inhibitory effects on cell viability, lactate dehydrogenase (LDH) and malondialdehyde (MDA) levels, cell apoptotic rate and caspase-3 activity. Importantly, HG-impaired angiogenesis was improved after netrin-1 overexpression by elevating cell migration, capillary-like tube formation and VEGF production. Mechanism assay substantiated that netrin-1 elevation increased the phosphorylation levels of AKT and eNOS, and NO production, which was notably suppressed by HG, indicating that netrin-1 overexpression restored HG-triggered impairment of the PI3K/AKT-eNOS pathway. More intriguingly, preconditioning with LY294002 (PI3K/AKT antagonist) or NG-monomethyl-I-arginine (eNOS inhibitor) antagonized netrin-1-induced activation of the PI3K/AKT-eNOS pathway. Concomitantly, treatment with these antagonists also attenuated the protective role of netrin- 1 in endothelial dysfunction upon HG stimulation. These results suggest that elevation of netrin-1 may restore HG-triggered impairment of HUVEC and angiogenesis by activating the PI3K/AKT-eNOS pathway, indicating a potential agent for wound healing in DFU patients.
\end{abstract}

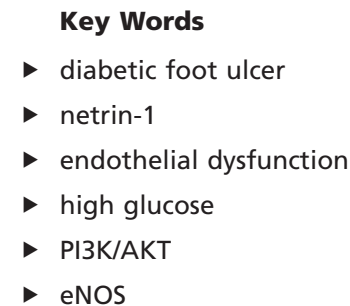

Journal of Molecular Endocrinology (2017) 58, 167-177
C 2017 Society for Endocrinology Printed in Great Britain
Published by Bioscientifica Ltd 


\section{Introduction}

Diabetes mellitus (DM) ranks as a complicated and chronic health disease and is commonly accepted to be the fifth common cause of death with the escalating incidence worldwide (Roglic et al. 2005). It is estimated that 366 million people suffered from diabetes in 2011, which is predicted to reach 522 million by 2030 . DM is recognized as a major contributor to high mortality and morbidity through a series of diabetic complications such as nephropathy and stroke. Among these, diabetic foot ulceration (DFU) is a proverbial consequence of DM, having 15\% prevalence among hospitalized DM patients (Woo et al. 2013). Patients with DFU suffer higher mortality and disability than patients without foot ulcers. DFU is characterized by lessened vascularity and angiogenesis and leads to $84 \%$ of diabetes-related lower leg amputations (Liu et al. 2014). Importantly, DFU represents a main contributor to non-traumatic lower limb amputation in developed countries, resulting in an annual cost of 300 million pounds (Posnett \& Franks 2008). Consequently, there is an urgent need to elucidate the mechanism underlying DFU and to develop efficient therapeutic strategies against DFU.

Hyperglycemia, a known characteristic of diabetes, usually results in refractory wound lesions and endothelial dysfunction, which, in turn, contributes to diabetic vascular complications including DFU (Marston \& Dermagraft 2006, Wu \& Chen 2016). It is generally believed that DFU is caused by neuropathy, infection and peripheral vascular disorders, which results in poor arterial inflow within limbs and consequently worse outcomes (Woo et al. 2013, Volmer-Thole \& Lobmann 2016). Impairment of angiogenesis induced by chronic hyperglycemia is a pivotal cause of delayed healing in DFU due to insufficient blood supply (Brem \& Tomic-Canic 2007, Carmeliet \& Jain 2011, Costa \& Soares 2013, Ruzehaji et al. 2014). The healing of diabetic wounds is a complex process, involving in various cellular processes and cytokines including NO (Brem \& Tomic-Canic 2007). Enhanced synthesis of NO and VEGF, both of which are critical regulators for vasculogenesis, can improve blood flow and, consequently, contribute to wound healing of diabetic foot ulcers (MohajeriTehrani et al. 2014). Recent study confirmed that elevating the pro-angiogenic response by attenuating flightless I obviously accelerated skin wound healing, implying a promising therapeutic strategy against DFU (Ruzehaji et al. 2014).
Netrin-1 is initially recognized to be an axon guidance molecule, and it has an analogous structure to laminin. Netrin-1 possesses a prominent role in a variety of physiological processes, including cell injury, proliferation and migration (Son etal.2013, Yang etal.2017). In umbilical cord blood-derived mesenchymal stem cells (UCB-MSC), netrin-1 protected against hypoxic injury-induced cell apoptosis via the expression of heat shock protein (HSP) 27 (Son et al. 2013). The growing body of evidence has corroborated a vital function of netrin-1 in injury repair of multiple diseases (Wang et al. 2008, Ke et al. 2014). Administration of netrin-1 antagonizes both ischemiareperfusion (I/R) injury of kidney and kidney function repair by suppressing endothelial cell activation and leukocyte infiltration (Wang et al. 2008). Emerging report demonstrated the reduction of plasma netrin-1 levels in patients diagnosed with type $2 \mathrm{DM}$, and netrin- 1 levels was found to be negatively related to insulin resistance and glucose homeostasis (Liu et al. 2016). Importantly, administration with netrin-1-expressing mesenchymal stem cells facilitated the repair of myocardial infarctiontriggered ischemic injury in diabetic mice by promoting NO production and subsequent neovessel formation (Ke et al. 2014). Netrin-1 also exerts the beneficial efficacy in the treatment of diabetic nephropathy (Mohamed et al. 2012, Tak et al. 2013). Unfortunately, its role and molecular mechanism in DFU are poorly elucidated. In this study, we aimed to investigate the levels of netrin-1 in DFU patients and hyperglycemia conditions in vitro. Furthermore, the role of netrin- 1 and its involvement in a potential mechanism of HUVEC dysfunction under high glucose were also investigated in the present research.

\section{Materials and methods}

\section{Patients}

Type 2 diabetic patients with DFU (DFU group, $n=35$ ) or not (DM group, $n=35$ ) and healthy individuals (control group, $n=30$ ) were recruited from the Department of Endocrinology, Xijing Hospital, Forth Military Medical University, during the period of August 2013-December 2014. Patients with DFU were enrolled into this research with Wagner classification DFU stages (2-4) and wound surface area over $1.5 \mathrm{~cm}^{2}$. All patients were matched for age and gender. Exclusion criteria for DFU patients included severe infection, hyperthyroidism, active

Published by Bioscientifica Ltd. 
osteomyelitis, skin diseases, pregnancy and kidney failure. Patients receiving any pharmaceutical treatment to enhance wound healing were also excluded. Individuals without a history of diabetes and with a normal fasting blood glucose levels were defined as the control group. All biochemical parameters were determined by routine techniques using a Hitachi-912 Autoanalyzer (Hitachi). This research was approved by the Ethics Committee of Xijing Hospital, Forth Military Medical University and conducted according to the Helsinki Declaration. All participants gave written informed consent.

\section{Detection of netrin-1 levels in plasma}

Participant blood samples were collected in EDTA containers and centrifuged at $503 \boldsymbol{g}$ for $10 \mathrm{~min}$. Then, plasma specimens were stored at $-80^{\circ} \mathrm{C}$ for subsequent experiments. The concentration of netrin- 1 in plasma specimens was measured according to the instruction of a commercial human netrin-1 ELISA kit (ToYong Bio, Shanghai, China).

\section{Cell culture and treatment}

HUVECs were purchased from ATCC. Cells were seeded in $75-\mathrm{cm}^{2}$ flasks and incubated with M199 medium (Grand Island, NY, USA) supplement with 10\% FBS. For glucose exposure, cells were cultured with M199 medium containing $5.5 \mathrm{mmol} / \mathrm{L}$ glucose (normal glucose group) or $33 \mathrm{mmol} / \mathrm{L}$ glucose (high glucose group) for $48 \mathrm{~h}$. All cells were maintained in a humidified incubator with $5 \% \mathrm{CO}_{2}$ at $37^{\circ} \mathrm{C}$.

\section{Assessment of netrin-1 mRNA levels by quantitative real-time PCR (qRT-PCR)}

Total RNA from cells was extracted using RNAiso Plus (TaKaRa Bio). Then, $1.5 \mu \mathrm{g}$ of extracted RNA was reverse transcribed to synthesize the cDNA with an ImProm II reverse-transcriptase kit (Promega). Afterward, $1 \mu \mathrm{L}$ of cDNA was used as a template for qRT-PCR assay using a SYBR Premix Ex TaqTM II Kit (TaKaRa). The specific primers for netrin-1 and $\beta$-actin were synthesized by Sangon (Shanghai, China) and used as follows: netrin-1, sense: 5'-TGGGGCGGTTGTCCTATTTC-3', antisense: $5^{\prime}$-GCCTTGTTGCTTGGCGTGT-3'; $\beta$-actin, sense: 5'-CACGATGGAGGGGCCGGACTCATC-3', anti-sense: 5'-TAAAGACCTCTATGCCAACACA GT-3'. The reaction was carried out using ABI PRISM Sequence Detector System 7500 (Perkin-Elmer, Applied Biosystems). The mRNA levels of netrin- 1 were normalized to $\beta$-actin. Relative mRNA levels were calculated using $2-\Delta \Delta C \mathrm{CT}$ method.

\section{Immunoblotting}

After lysis in RIPA lysis buffer, the extracted protein was separated by $12 \%$ SDS-PAGE and then electroblotted onto PVDF membranes (Millipore). For Western blotting assay, the membrane was blocked with 5\% skim milk for $2 \mathrm{~h}$. After rinsing, primary antibodies were added and incubated at $4^{\circ} \mathrm{C}$ overnight, including antibodies against netrin-1 (1:3000) (Abcam), phosphorylated (p)-AKT and AKT (1:1000) (Cell Signaling Technology), p-eNOS (1:600, Abcam), eNOS (1:600, Abcam), VEGF (1:100) (Invitrogen) and $\beta$-actin (1:500, Invitrogen). Then, the goat anti-rabbit $(1: 500)$ and anti-mouse $(1: 10,000)$ secondary antibodies conjugated with HRP (Invitrogen) were added and incubated at room temperature for $1 \mathrm{~h}$. To visualize the immunoreactive proteins, ECL reagent (Invitrogen) was added. The binding bands were analyzed using a Gel DocTM XR imaging system (Bio-Rad) and quantified via Quantity One software (Bio-Rad). The relative protein expression was normalized to $\beta$-actin.

\section{Recombinant adenovirus vector construction and transduction}

To construct a recombinant adenovirus vector expressing netrin-1, full-length cDNA of human netrin-1 was inserted into pAdTrack-CMV (Agilent) plasmidcontaining green fluorescent protein (GFP). After homologous recombination with pAdEasy-1 in BJ5183, E. coli strain, DNA sequencing was carried out to evaluate the insert identity and orientation by Sangon Company. The Ad-netrin-1 vectors were then transfected into HEK293T cells (ATCC). After propagation in 293T cells, the amplified virus was purified using $\mathrm{CsCl} 2$ gradient centrifugation. Then, the viral titer was assessed using the Adeno-X Rapid Titer kit (BD Biosciences, San Jose, CA, USA). For transduction, HUVECs were infected with the recombinant virus suspension of Ad-Srx-1, or Ad-GFP, at the titer of $1 \times 10^{9} \mathrm{TU} / \mathrm{mL}$ for $48 \mathrm{~h}$ at $37^{\circ} \mathrm{C}$.

\section{Cell proliferation assay by MTT}

Cells were seeded into 96-well plates at a density of 5000 cells/well. Then, cells were treated with either eNOS inhibitor $N^{\mathrm{G}}$-monomethyl-L-arginine $(\mathrm{NMA}, 100 \mu \mathrm{M})$ or PI3K/AKT inhibitor LY294002 $(20 \mu \mathrm{M})$ (Sigma) prior to infection with Ad-netrin-1 under high glucose. After that,

Published by Bioscientifica Ltd 
$20 \mu \mathrm{L}$ of MTT ( $5 \mathrm{mg} / \mathrm{mL}$, Sigma) was added to each well for a further 4-h incubation. Subsequently, formazan crystals were dissolved by DMSO for $10 \mathrm{~min}$. Cell viability was then assessed by detecting the absorbance at $570 \mathrm{~nm}$ (OD570) and expressed by calculating the absorbance percentage of the treated group vs control group.

\section{Detection of lactate dehydrogenase (LDH) and malondialdehyde (MDA) levels}

After the collection of culture medium, the activities of LDH and MDA were measured as per the manufacturer's protocols of LDH and MDA assay kits (Nanjing Jiancheng Bioengineering Institute, Nanjing, China).

\section{Caspase-3 assay}

The activity of caspase-3 was detected for different groups using the caspase-3 Fluorescent Assay Kits (Clontech). Cells under the indicated treatment were lysed and centrifuged. Then, the harvested supernatants were incubated for $1 \mathrm{~h}$ with $5 \mu \mathrm{L}$ of DEVD-AFC, a specific substrate for caspase-3. Finally, caspase-3 activity was evaluated by measuring the fluorescence intensities using a FL600 fluorescent reader (BioTek Instruments).

\section{Apoptosis analysis}

Cell apoptotic rates were measured by flow cytometric analysis using Annexin V-FITC/PI staining. Briefly, cells in different groups were trypsinized. After rinsing with PBS, cells were suspended in binding buffer, and then incubated with $10 \mu \mathrm{L}$ Annexin V-FITC (Beyotime, Shanghai, China) for 15 min under darkness. Then, $5 \mu \mathrm{L}$ of PI was also added for an additional $5 \mathrm{~min}$. The specimens were subjected into FACSCalibur flow cytometer (BECTON, Franklin Lakes, NJ, USA) to determine the apoptotic rates.

\section{Detection of cell migration by Transwell assay}

The migration of HUVECs under various treatment specimens was evaluated using a Transwell system (Millipore) with $8 \mu \mathrm{m}$ pore size. For migration, cells $\left(2 \times 10^{4}\right)$ were seeded into the upper chamber with serum-free M199 medium, and chemotaxis was performed by loading 10\% FBS to the lower chamber. After approximately $24 \mathrm{~h}$, cells that had migrated to the bottom surface of membrane were fixed with $4 \%$ paraformaldehyde, followed by staining with $0.1 \%$ crystal violet. The mean number of migrated cells was calculated from five random fields per specimen using a BX51 microscope (Olympus).

\section{Tube formation in vitro}

The formation of capillary tube was assessed by Matrigel analysis. After seeding in 96-well plates pre-coated with Matrigel (BD Biosciences), cells $\left(1 \times 10^{4} /\right.$ well) were treated with the indicated conditions. Twenty-four hours later, the formation of capillary-like tubular structures were captured under an inverted microscope. The number of tubes was quantified by assessing five randomly selected fields and presented as the percentage of tubes formed in control group.

\section{ELISA assay of VEGF levels}

After treatment with indicated conditions, the culture medium was harvested and centrifuged. Then, VEGF levels released into supernatants were determined using a Human VEGF Quantikine ELISA Kit (R\&D Systems) in accordance with the manufacturer's instructions.

\section{Measurement of NO production}

NO production in different culture medium was evaluated by determining the stable metabolite of NO, nitrite. After 10-min incubation with Griess reagent containing 1\% sulfanilamide, 2\% phosphoric acid and 0.1\% naphthyl ethylenediamine dihydrochloride, samples were then analyzed by a SpectraMax Plus 384 spectrophotometer (Molecular Device, Sunnyvale, CA, USA) at $540 \mathrm{~nm}$. Nitrite contents were assessed by reference to standard curves of nitrite.

\section{Statistical analysis}

All data were obtained from at least three independent trials and analyzed by SPSS 19.0 software. Results were exhibited as mean \pm standard deviation (s.D.). Statistical significances $(P<0.05$ and $P<0.01)$ were evaluated by Student's $t$-test for two groups and one-way ANOVA for three or more groups, followed by a post hoc least significant difference (LSD) test.
() 2017 Society for Endocrinology Printed in Great Britain
Published by Bioscientifica Ltd 
Table 1 Characteristics of patients.

\begin{tabular}{|c|c|c|c|}
\hline & $\begin{array}{c}\text { Healthy } \\
\text { control }(n=30)\end{array}$ & DM $(n=35)$ & DFU $(n=35)$ \\
\hline Age (years) & $52.4 \pm 2.1$ & $54.3 \pm 2.6$ & $55.2 \pm 3.1$ \\
\hline \multicolumn{4}{|l|}{ Gender } \\
\hline Male & 17 & 16 & 18 \\
\hline Female & 13 & 19 & 17 \\
\hline Weight (kg) & $62.18 \pm 5.79$ & $64.26 \pm 6.17$ & $65.11 \pm 5.87$ \\
\hline $\begin{array}{l}\text { Fasting plasma } \\
\text { glucose } \\
\text { (mmol/L) }\end{array}$ & $5.14 \pm 0.64$ & $9.87 \pm 2.89 *$ & $10.02 \pm 2.51$ * \\
\hline $\mathrm{HbA} 1 \mathrm{c}(\%)$ & $4.06 \pm 0.38$ & $8.76 \pm 0.41 *$ & $8.25 \pm 0.53^{*}$ \\
\hline $\begin{array}{l}\text { Serum } \\
\text { triglyceride } \\
(\mathrm{mmol} / \mathrm{L})\end{array}$ & $7.96 \pm 3.11$ & $8.43 \pm 3.32$ & $8.22 \pm 3.54$ \\
\hline $\begin{array}{l}\text { Total cholesterol } \\
(\mathrm{mmol} / \mathrm{L})\end{array}$ & $9.53 \pm 2.14$ & $8.76 \pm 2.87$ & $8.84 \pm 3.06$ \\
\hline
\end{tabular}

\section{Results}

\section{Decreased expression of netrin-1 in patients with DFU and HUVECs under HG}

As shown in Table 1, no differences were observed in the clinical and biochemical characterizations of patient subjects, excepting the higher concentrations of fasting plasma glucose and HbA1c in the DM and DFU groups. Further analysis corroborated an obvious downregulation of netrin-1 levels in plasma specimens taken from DM and DFU patients, compared to that in control group (Fig. 1A). Importantly, netrin-1 was presented at the lower content in DFU group relative to DM group. Moreover, the dramatical decreases in netrin-1 mRNA (Fig. 1B) and protein levels (Fig. 1C) were also demonstrated in HUVECs upon HG exposure in contrast to normal glucose-treated group. Together, these results suggested a potential role of netrin-1 in the progression of DFU.

\section{Elevation of netrin-1 antagonizes HUVEC injury triggered by HG exposure}

To clarify the effect of netrin-1 on vascular endothelial cell injury under HG condition, cells were infected with Ad-netrin-1. The upregulation of netrin-1 in Ad-netrin-1 group was validated by qRT-PCR and Western assay (Fig. 2A and B). Functional analysis manifested that HG exposure reduced HUVEC viability to $40.11 \%$, whereas this inhibitory function was notably attenuated after netrin-1 upregulation (Fig. 2C). Additionally, administration with HG augmented LDH release (Fig. 2D) and MDA levels (Fig. 2E), both the indices of cell injury. Intriguingly, ectopic expression of netrin-1 restrained the increases in LDH and MDA levels. Simultaneously, elevation of netrin-1 further attenuated the apoptotic rates of HUVECs upon $\mathrm{HG}$ from $32.67 \%$ to $12.78 \%$ (Fig. 2F). Furthermore, the high activity of caspase-3, a widely used indicator of cell apoptosis, in HUVECs under HG was also dramatically suppressed by netrin-1 overexpression (Fig. 2G). These data indicated that netrin-1 could protect against high glucose-induced HUVEC injury.

\section{Ectopic expression of netrin-1 restores HG-impaired angiogenesis in HUVECs}

The numbers of migrated cells were significantly reduced in response to HG stimulation (Fig. 3A). However,
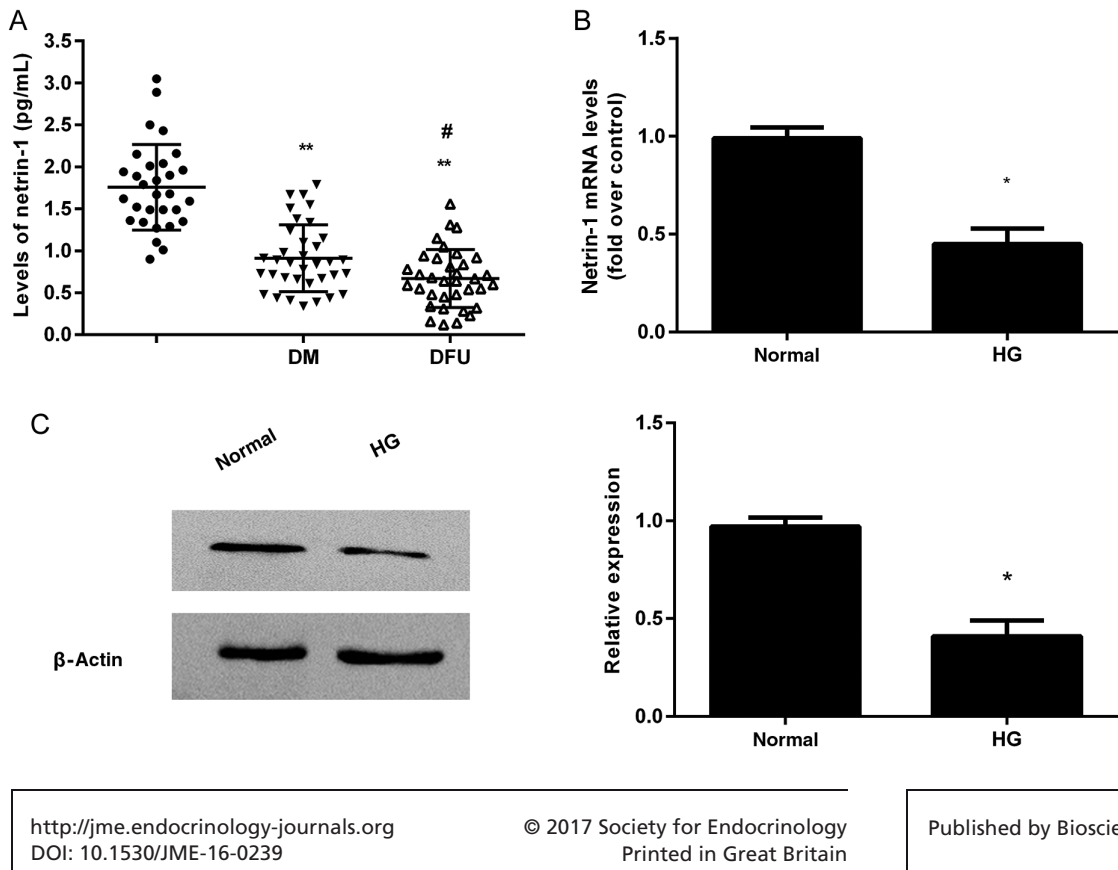

http://jme.endocrinology-journals.org DOI: 10.1530/JME-16-0239
๑) 2017 Society for Endocrinology Printed in Great Britain
Figure 1

Downregulation of netrin-1 in patients with DFU and HUVECs upon HG exposure. (A) Plasma samples were collected from health control, DM and DFU patients. The levels of netrin-1 in plasma were determined by ELISA assay. ${ }^{* *} P<0.01$ vs control groups. $\# P<0.05$ vs DM groups. (B) HUVECs were exposed to normal glucose, or HG, for $48 \mathrm{~h}$, the mRNA levels of netrin- 1 were evaluated using qRT-PCR. (C) The protein levels of netrin- 1 in normal and HG groups. ${ }^{*} P<0.05$ vs normal glucose group. 
A

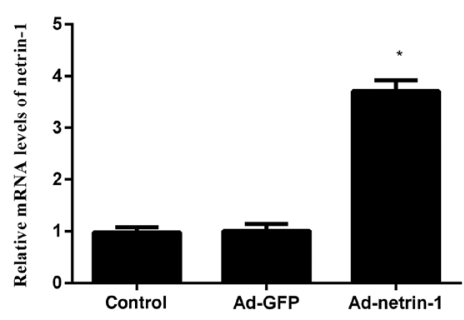

D

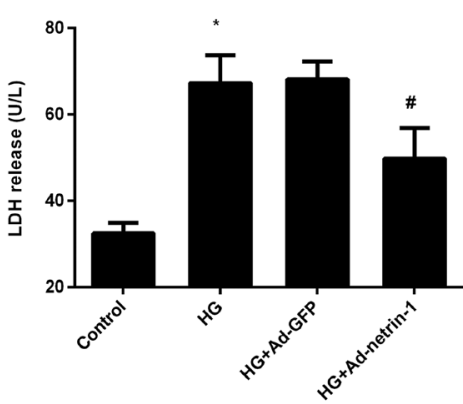

B

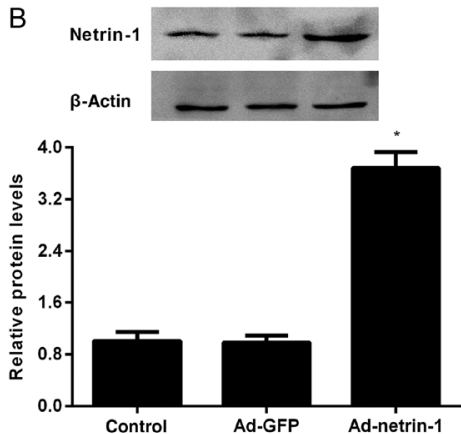

E

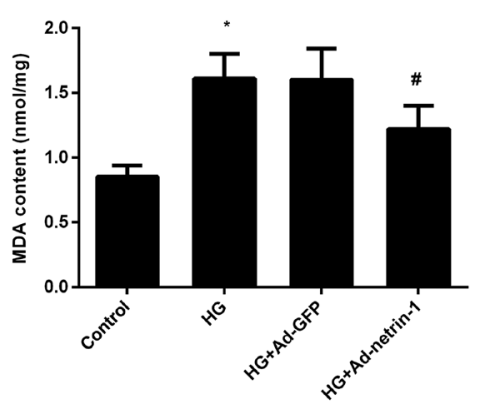

C

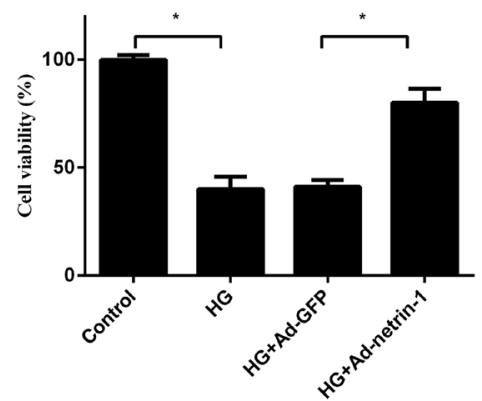

G

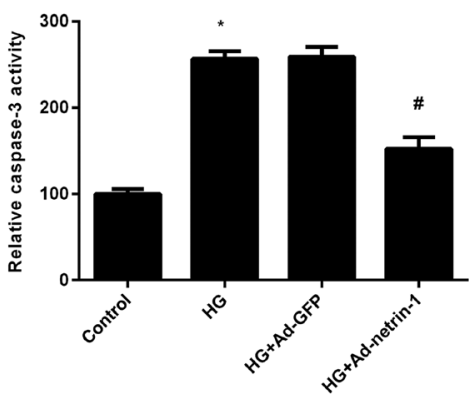

F
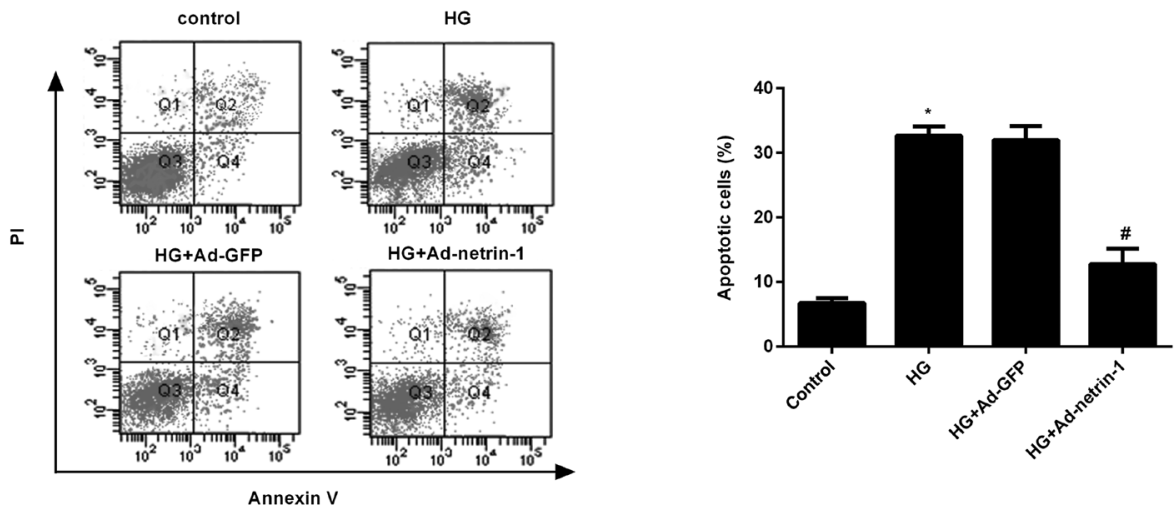

Figure 2

Upregulation of netrin-1 protected against HUVEC injury by HG. HUVECs were infected with Ad-netrin-1, or AD-GFP, prior to subject to HG. (A and B) Expression levels of netrin-1 mRNA (A) and protein (B) were detected independently by qRT-PCR and Western blotting. (C) MTT assay was performed to assess the cell viability. (D and E) Effect of netrin-1 overexpression on LDH leakage (D) and MDA concentration (E) triggered by HG. (F) After dual-

staining with Annexin $V$ and Pl, cell apoptotic rate was measured by flow cytometry. (G) Caspase-3 activity was measured by fluorometric assay. ${ }^{*} P<0.05$.

the inhibitory role of $\mathrm{HG}$ was reversed after netrin-1 overexpression. Importantly, upregulation of netrin-1 attenuated the inhibitory effect of HG on capillarylike tube formation by HUVECs (Fig. 3B). Moreover, exposure of HUVECs to HG dampened the VEGF expression, a critical factor in angiogenesis, which was noticeably ameliorated after netrin-1 elevation (Fig. 3C). Concomitantly, the decreased concentrations of VEGF in medium under HG stimulation were alleviated when cells were infected with Ad-netrin-1 vectors (Fig. 3D). These observations manifested that elevation of netrin-1 could execute the protective response to $\mathrm{HG}$-injured angiogenesis in HUVECs.

Netrin-1 upregulation restores the injury in the activation of PI3K/AKT-eNOS pathway upon HG stimulation

To elucidate the underlying mechanism for the beneficial role of netrin-1 upregulation in endothelial dysfunction under $\mathrm{HG}$, the activation of PI3K/AKTeNOS signaling was explored. Here, exposure to HG reduced the phosphorylation level of AKT, but not in the 
A

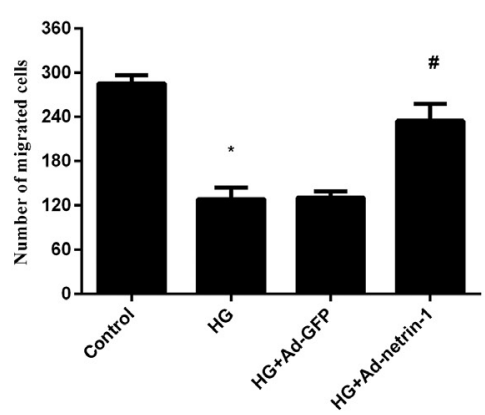

C

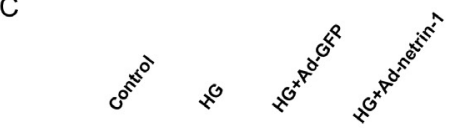

$\beta$-Actin

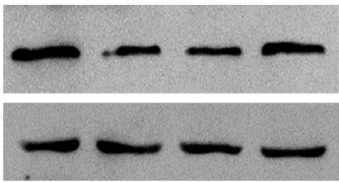

B

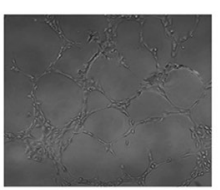

HG+Ad-GFP
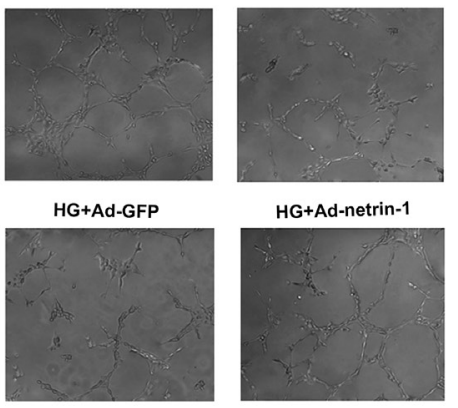

HG+Ad-netrin-1
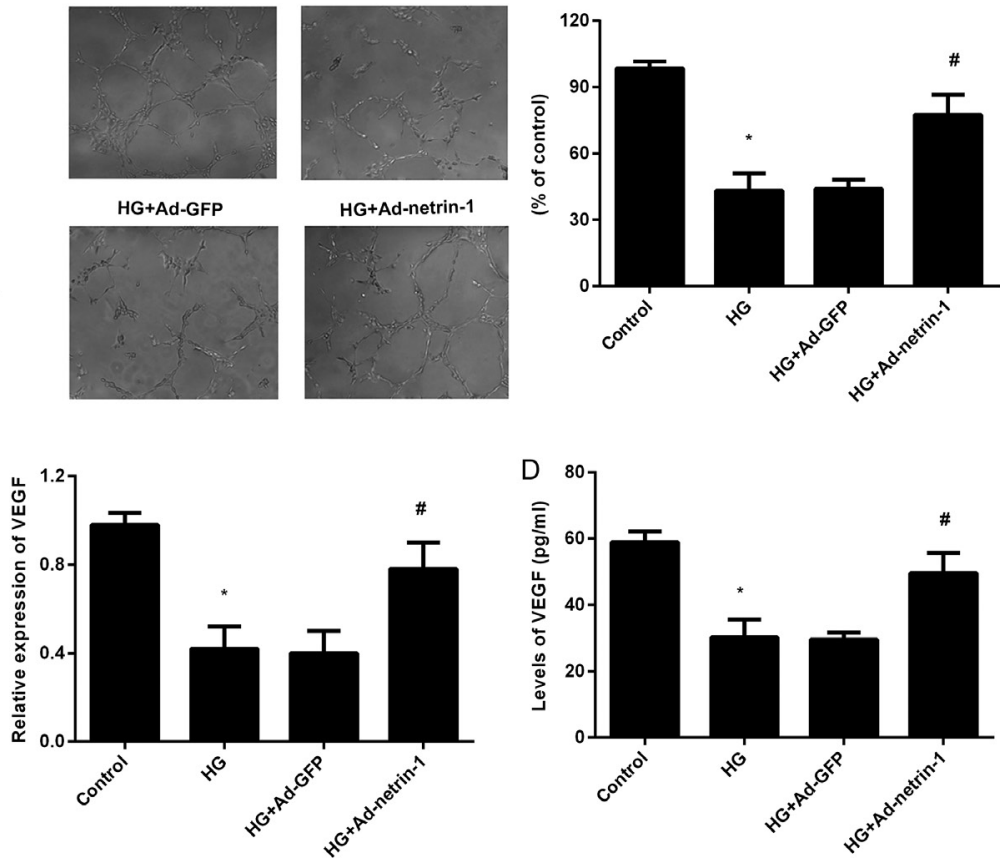

Figure 3

Effect of netrin-1 elevation on the impairment of angiogenesis in response to HG. After infection with Ad-netrin-1, HUVECs were incubated with HG. (A) Cell migration was analyzed by Transwell chamber. (B) Effect on capillary-like tube formation was evaluated by culturing on Matrigel (magnification $\times 100$ ). (C) The expression of VEGF protein was determined by Western blotting assay. (D) Upregulation of netrin-1 ameliorated the inhibitory role of HG in VEGF concentration in culture medium. ${ }^{*} P<0.05$ vs control groups. ${ }^{*} P<0.05$ vs $\mathrm{HG}$ groups.

A
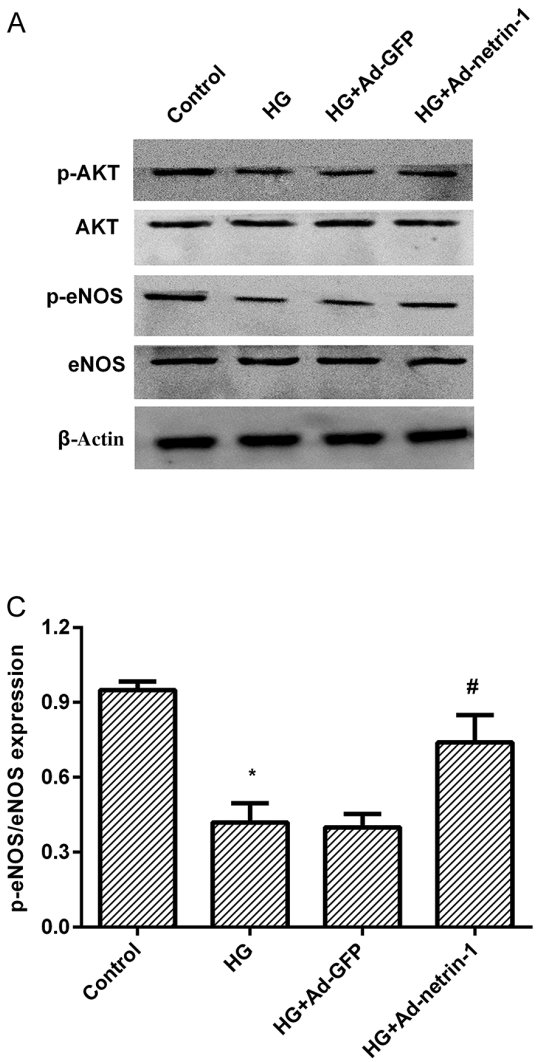

B

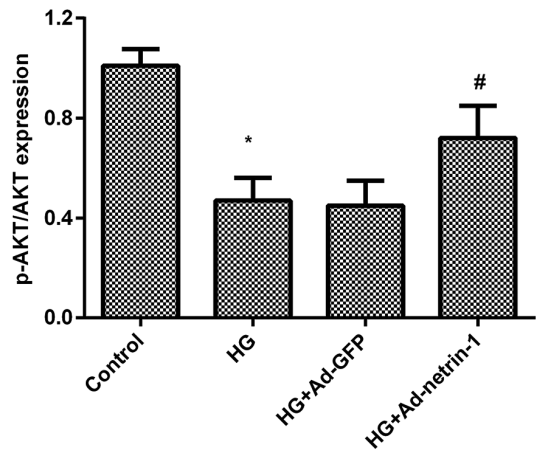

D

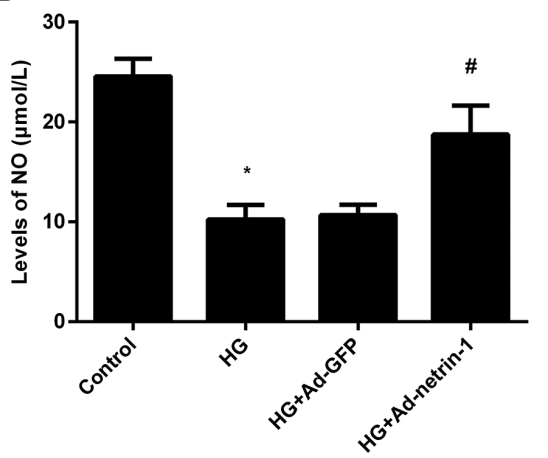

Figure 4

Netrin-1 restored the impairment of the PI3K/ AKT-eNOS pathway in HG-stimulated HUVECs. (A) Phosphorylation of AKT and eNOS was determined in HG-treated cells that were infected with Ad-netrin-1, or not, by Western blotting.

(B) Quantification analysis of $\mathrm{p}$-AKT/AKT protein.

(C) Quantification analysis of p-eNOS/eNOS

expression. (D) Effects of netrin-1 on NO concentration in the indicated groups. $* P<0.05$ vs control groups. $\# P<0.05$ vs HG groups. 
A

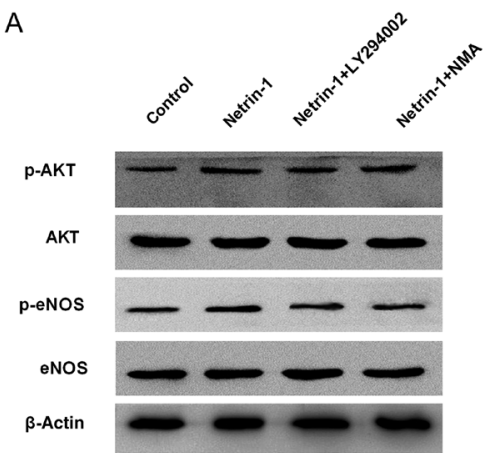

D

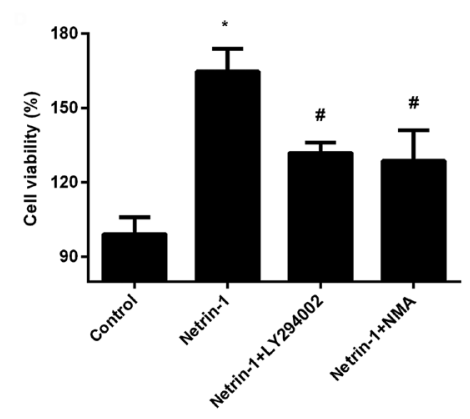

B

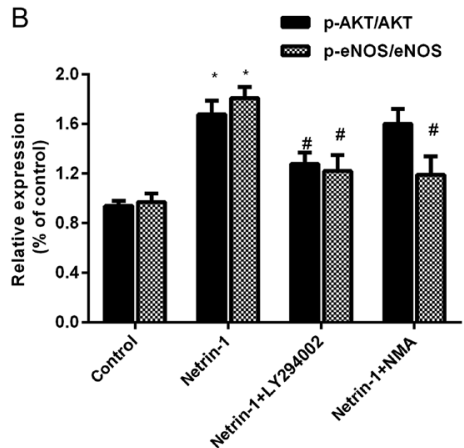

C
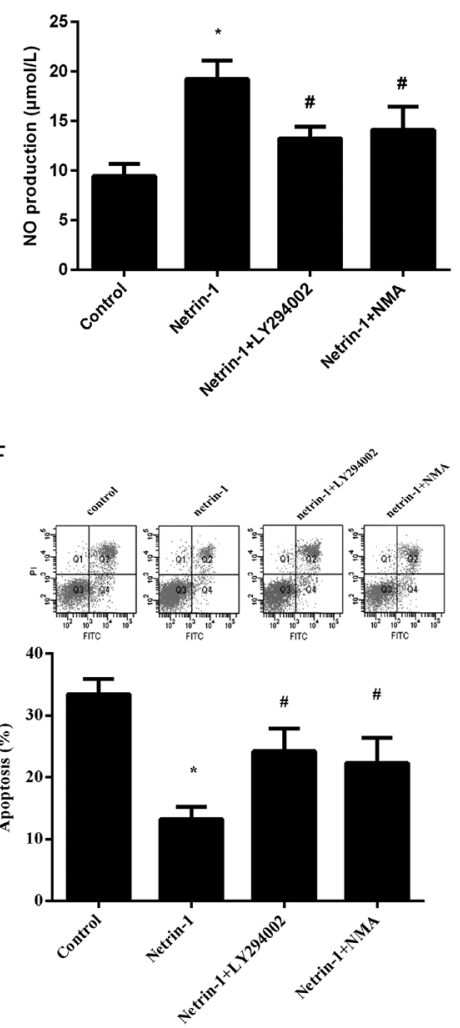

E

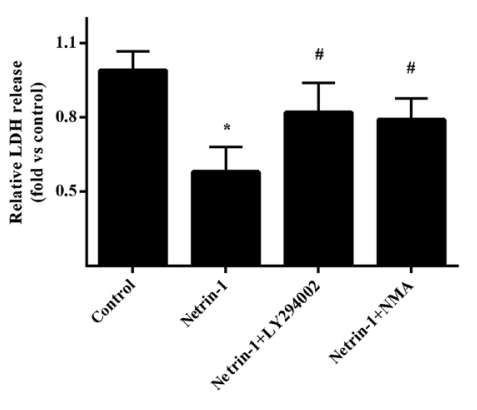

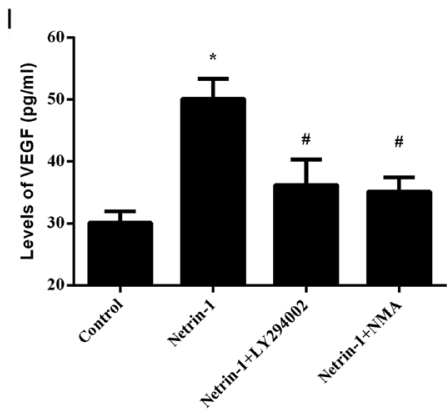

Figure 5

The PI3K/AKT-eNOS signaling was involved in the protective role of netrin-1 in endothelial dysfunction. (A) After preconditioning with LY294002, or NMA, the activation of PI3K/AKT-eNOS pathway in netrin-1-overexpressed cells upon HG was analyzed by Western blotting. (B) The corresponding quantified assay of protein expression. (C) Pretreatment with LY294002, or NMA, attenuated the production of NO induced by netrin-1. (D, E, F, G, $\mathrm{H}$ and I) The corresponding effects on cell viability (D), LDH leakage (E), cell apoptosis (F), cell migration (G), tube formation (H) (magnification $\times 100)$ and VEGF production (I). ${ }^{*} P<0.05$ vs control groups. ${ }^{\#} P<0.05$ vs netrin-1-treated groups.

expression of AKT protein (Fig. 4A). Simultaneously, the protein expression of p-eNOS was also suppressed under HG, whereas without the change in eNOS expression. However, upregulation of netrin-1 dramatically increased the phosphorylation levels of AKT and eNOS, but had little effects on the total AKT and eNOS expression (Fig. 4B and $\mathrm{C}$ ), indicating that netrin-1 elevation restored the impairment of PI3K/AKT-eNOS signaling induced by HG. Additionally, HG exposure decreased the concentration of NO production from $24.56 \mu \mathrm{mol} / \mathrm{L}$ to $10.23 \mu \mathrm{mol} / \mathrm{L}$, which was increased into $18.74 \mu \mathrm{mol} / \mathrm{L}$ when netrin-1 was overexpressed in HUVECs (Fig. 4D). 


\section{Netrin-1 improves HUVEC dysfunction in response to HG through the PI3K/AKT-eNOS pathway}

We further investigated whether the PI3K/AKT-eNOS pathway was responsible for the protective effects of netrin-1 on HG-triggered cell and angiogenesis impairment. As shown in Fig. 5A and B, prior administration with LY294002 mitigated the augment in the expression of p-AKT and p-eNOS induced by netrin-1 overexpression. Concomitantly, pretreatment with NMA, an antagonist of the eNOS pathway, also attenuated netrin-1-increased phosphorylation levels of eNOS. The augmented production of $\mathrm{NO}$ in netrin-1-overexpressed groups was consistently decreased in HG-treated cells after pretreatment with both LY294002 and NMA (Fig. 5C). Moreover, ectopic expression of netrin-1 elevated cell viability upon $\mathrm{HG}$, which was decreased when cells were preconditioned with LY294002 and NMA (Fig. 5D). At the same time, blocking the PI3K/AKT and eNOS pathways with their respective antagonists also potentiated the release of LDH (Fig. 5E) and cell apoptosis (Fig. 5F), which was attenuated by netrin-1 overexpression. Further, functional assays documented that netrin-1-induced increases in migrated cell number (Fig. 5G) and tube formation (Fig. 5H) were both inhibited in LY294002- and NMA-pretreated groups. Additionally, preconditioning with LY294002 and NMA also antagonized the production of VEGF triggered by netrin-1 elevation (Fig. 5I).

\section{Discussion}

Hyperglycemia is a predominantly metabolic feature of diabetes and can trigger various macrovascular and microvascular-related complications. As a common and serious chronic complication of diabetes, DFU remains an unsolved health problem due to the high mortality and disability rates, with a poignant amputation being performed approximately every $30 \mathrm{~s}$ worldwide (Boulton et al. 2005). To develop a new and effective strategy to treat DFU is urgently needed. Decreased levels of netrin-1 had been observed in diabetic patients (Liu et al. 2016). Importantly, increasing evidences have corroborated the protective role of netrin-1 expression against diabetes-induced complications such as diabetic nephropathy and myocardial infarction (Tak et al. 2013, Ke et al. 2014). To date, no data are available on its function in diabetes-induced DFU. Coincident with a previous research (Liu et al. 2016), an obvious downregulation of netrin-1 in plasma was observed in diabetic patients in contrast to healthy controls. More intriguingly, lower levels of netrin-1 were detected in patients with DFU relative to those without DFU, indicating a potential critical role of netrin-1 for wound healing in DFU.

Endothelial dysfunction in diabetes is a pivotal characteristic of diabetes-mediated vascular complications including DFU (Gerasimchuk et al. 2014, Dong et al. 2016, Suganya et al. 2016). Once impaired by hyperglycemia, vascular endothelial cells will lose their ability to regulate blood flow, nutrient delivery and angiogenesis, and this, in turn, can affect wound healing in diabetic patients (Marston \& Dermagraft 2006). Netrin-1 has recently attracted increasing interests in wound repair progression in diabetic complications. Previous researches have revealed a protective role of netrin-1 against ischemia/ reperfusion kidney injury and cardiac injury (Tak et al. 2013, Siu et al. 2015). Importantly, ectopic expression of netrin-1 exerts a benefit effect on repair impairment in diabetes-induced myocardial infarction and diabetic nephropathy, making it as a potentially promising agent against diabetic complication (Tak et al. 2013, Ke et al. 2014). To further elucidate the role of netrin-1 in DFU, we investigated its function in vascular endothelial cell dysfunction under high glucose exposure. As expected, high glucose treatment inhibited netrin-1 expression in HUVECs. Importantly, upregulation of netrin-1 ameliorated high glucose-inhibited cell viability and suppressed high glucose-increased cell apoptosis. Simultaneously, the release of LDH induced by high glucose, a marker for cell injury, was also reduced after netrin-1 overexpression concomitant with MDA decrease, an indicator to estimate reactive oxygen species (ROS)induced cell injury upon high glucose. These results suggest that netrin-1 elevation may protect against HUVEC injury in response to high glucose.

Impaired vasculogenesis is widely recognized to be a key contributor to delayed wound healing (Ruzehaji et al. 2014, Zhou et al. 2017). We, therefore, next investigated the angiogenic function of netrin-1 in HUVECs stimulated with high glucose. Intriguingly, ectopic expression of netrin-1 restored the inhibitory effects of high glucose on cell migration and tube formation. Concomitantly, netrin-1 also elevated the production of VEGF, a proangiogenic cytokine, under high glucose. A previous study also proved an essential role of netrin-1 in angiogenesis of rat placenta (Xie et al. 2011). Analogously, netrin-1 expression can improve capillary formation and blood vessel density in mice with diabetic myocardial infarction (Ke et al. 2014). Hence, these observations provoke us to conclude that overexpression of netrin-1 may facilitate wound healing of DFU by ameliorating endothelial

Published by Bioscientifica Ltd. 
dysfunction by restoring the impairment of cell growth and vascularization.

Cumulatively, abundant researchers agree that the PI3K/AKT pathway possesses a critical role in wound healing under diverse pathological conditions including hyperglycemia (Liu et al. 2014, Zhu et al. 2014). Once activated, the phosphorylation of AKT can directly phosphorylate eNOS and induce the subsequent production of NO (Yao et al. 2016). eNOS convincingly acts as a pivotal regulator of vascularization by mediating NO generation. To clarify the molecular mechanism involved in the protective role of netrin-1 against high glucose-induced endothelial dysfunction, we explored the activation of PI3K/AKT-eNOS signaling. Within agreement with this hypothesis, elevation of netrin-1 attenuated the decrease in the phosphorylation of AKT and eNOS when exposed to high glucose, as well as increased NO levels. As a critical and potent vasodilator, NO released by endothelia can maintain vascular integrity of normal blood vessels and its decrease results in vascular malfunction during the progression of insulin resistance (Zhu et al. 2014). Moreover, the deficiency of NO accounts for the damaged vascular regeneration in diabetic ischemic injury animal model (Yan et al. 2009). Further analysis confirmed that blocking the PI3K/AKT or eNOS pathway attenuated the restored potential of netrin-1 elevation in cell growth and pro-angiogenic impairment under conditions of high glucose exposure.

Collectively, this study demonstrated the decrease of netrin-1 levels in patients with DFU. More importantly, overexpression of netrin-1 could antagonize endothelial dysfunction by restoring cell injury and impaired angiogenesis upon high glucose exposure by regulating the PI3K/AKT-eNOS signaling. Therefore, this study highlights netrin-1 as a potentially promising agent for wound healing of diabetic vascular complications, including DFU. Emerging studies have verified the critical role of augmented inflammatory response in the pathological process of diabetic vascular complications (Tuttolomondo et al. 2015, Afarideh et al . 2016, Wu \& Chen 2016). Recently, several studies have corroborated that netrin-1 exerts the critical roles in the progression of inflammatory diseases (Podjaski et al. 2015, Mediero et al. 2016). Accordingly, further studies will focus on the effect of netrin-1 on the inflammatory response to hyperglycemia in vitro and DFU model.

\section{Declaration of interest}

The authors declare that there is no conflict of interest that could be perceived as prejudicing the impartiality of the research reported.

\section{Funding}

This work did not receive any specific grant from any funding agency in the public, commercial, or not-for-profit sector.

\section{References}

Afarideh M, Ghanbari P, Noshad S, Ghajar A, Nakhjavani M \& Esteghamati A 2016 Raised serum 25-hydroxyvitamin D levels in patients with active diabetic foot ulcers. British Journal of Nutrition 115 1938-1946. (doi:10.1017/S0007114516001094)

Boulton AJ, Vileikyte L, Ragnarson-Tennvall G \& Apelqvist J 2005 The global burden of diabetic foot disease. Lancet 366 1719-1724. (doi:10.1016/S0140-6736(05)67698-2)

Brem H \& Tomic-Canic M 2007 Cellular and molecular basis of wound healing in diabetes. Journal of Clinical Investigation 117 1219-1222. (doi:10.1172/JCI32169)

Carmeliet P \& Jain RK 2011 Molecular mechanisms and clinical applications of angiogenesis. Nature 473 298-307. (doi:10.1038/ nature10144)

Costa PZ \& Soares R 2013 Neovascularization in diabetes and its complications. Unraveling the angiogenic paradox. Life Sciences $\mathbf{9 2}$ 1037-1045. (doi:10.1016/j.lfs.2013.04.001)

Dong Y, Wu Y, Choi HC \& Wang S 2016 Diabetic endothelium dysfunction, cardiovascular complications, and therapeutics. Journa of Diabetes Research 20165349801. (doi:10.1155/2016/5349801)

Gerasimchuk PA, Kisil PV, Vlasenko VG \& Pavlyshin AV 2014 Endothelial dysfunction indicators in patients with diabetic foot syndrome. Vestnik Rossiiskoi Akademii Meditsinskikh Nauk 69(5-6) 107-110. (doi:10.15690/vramn.v69i5-6.1053)

Ke T, Wu Y, Li L, Liu Y, Yao X, Zhang J, Kong D \& Li C 2014 Netrin-1 ameliorates myocardial infarction-induced myocardial injury: mechanisms of action in rats and diabetic mice. Human Gene Therapy 25 787-797. (doi:10.1089/hum.2014.021)

Liu M, Xiang G, Lu J, Xiang L, Dong J \& Mei W 2014 TRAIL protects against endothelium injury in diabetes via Akt-eNOS signaling. Atherosclerosis 237 718-724. (doi:10.1016/j.atherosclerosis. 2014.10.013)

Liu C, Ke X, Wang Y, Feng X, Li Q, Zhang Y, Zhu J \& Li Q 2016 The level of netrin-1 is decreased in newly diagnosed type 2 diabetes mellitus patients. BMC Endocrine Disorders 16 33. (doi:10.1186/ s12902-016-0112-z)

Marston WA \& Dermagraft Diabetic Foot Ulcer Study Group 2006 Risk factors associated with healing chronic diabetic foot ulcers: the importance of hyperglycemia. Ostomy Wound Management 52 26-28, 30, 32 passim.

Mediero A, Wilder T, Ramkhelawon B, Moore KJ \& Cronstein BN 2016 Netrin-1 and its receptor Unc5b are novel targets for the treatment of inflammatory arthritis. FASEB Journal 30 3835-3844. (doi:10.1096/ fj.201600615R)

Mohajeri-Tehrani MR, Nasiripoor F, Torkaman G, Hedayati M, Annabestani Z \& Asadi MR 2014 Effect of low-intensity direct current on expression of vascular endothelial growth factor and nitric oxide in diabetic foot ulcers. Journal of Rehabilitation Research and Development 51 815-824. (doi:10.1682/JRRD.2013.08.0174)

Mohamed R, Jayakumar C, Ranganathan PV, Ganapathy V \& Ramesh G 2012 Kidney proximal tubular epithelial-specific overexpression of http://jme.endocrinology-journals.org

DOI: 10.1530/JME-16-0239
(C) 2017 Society for Endocrinology Printed in Great Britain
Published by Bioscientifica Ltd 
netrin-1 suppresses inflammation and albuminuria through suppression of COX-2-mediated PGE2 production in streptozotocininduced diabetic mice. American Journal of Pathology 181 1991-2002. (doi:10.1016/j.ajpath.2012.08.014)

Podjaski C, Alvarez JI, Bourbonniere L, Larouche S, Terouz S, Bin JM, Lecuyer MA, Saint-Laurent O, Larochelle C, Darlington PJ, et al. 2015 Netrin 1 regulates blood-brain barrier function and neuroinflammation. Brain 138 1598-1612. (doi:10.1093/brain/awv092)

Posnett J \& Franks PJ 2008 The burden of chronic wounds in the UK. Nursing Times 104 44-45.

Roglic G, Unwin N, Bennett PH, Mathers C, Tuomilehto J, Nag S, Connolly V \& King H 2005 The burden of mortality attributable to diabetes: realistic estimates for the year 2000. Diabetes Care $\mathbf{2 8}$ 2130-2135. (doi:10.2337/diacare.28.9.2130)

Ruzehaji N, Kopecki Z, Melville E, Appleby SL, Bonder CS, Arkell RM, Fitridge R \& Cowin AJ 2014 Attenuation of flightless I improves wound healing and enhances angiogenesis in a murine model of type 1 diabetes. Diabetologia 57 402-412. (doi:10.1007/s00125-013-3107-6)

Siu KL, Lotz C, Ping P \& Cai H 2015 Netrin-1 abrogates ischemia/ reperfusion-induced cardiac mitochondrial dysfunction via nitric oxide-dependent attenuation of NOX4 activation and recoupling of NOS. Journal of Molecular and Cellular Cardiology 78 174-185. (doi:10.1016/j.yjmcc.2014.07.005)

Son TW, Yun SP, Yong MS, Seo BN, Ryu JM, Youn HY, Oh YM \& Han HJ 2013 Netrin-1 protects hypoxia-induced mitochondrial apoptosis through HSP27 expression via DCC- and integrin alpha6beta4dependent Akt, GSK-3beta, and HSF-1 in mesenchymal stem cells. Cell Death and Disease 4 e563. (doi:10.1038/cddis.2013.94)

Suganya N, Bhakkiyalakshmi E, Sarada DV \& Ramkumar KM 2016 Reversibility of endothelial dysfunction in diabetes: role of polyphenols. British Journal of Nutrition 116 223-246. (doi:10.1017/ S0007114516001884)

Tak E, Ridyard D, Badulak A, Giebler A, Shabeka U, Werner T, Clambey E, Moldovan R, Zimmerman MA, Eltzschig HK, et al. 2013 Protective role for netrin-1 during diabetic nephropathy. Journal of Molecular Medicine 91 1071-1080. (doi:10.1007/s00109013-1041-1)

Tuttolomondo A, Maida C \& Pinto A 2015 Diabetic foot syndrome: immune-inflammatory features as possible cardiovascular markers in diabetes. World Journal of Orthopedics 6 62-76. (doi:10.5312/wjo.v6.i1.62)
Volmer-Thole M \& Lobmann R 2016 Neuropathy and diabetic foot syndrome. International Journal of Molecular Sciences $\mathbf{1 7}$ E917. (doi:10.3390/ijms17060917)

Wang W, Reeves WB \& Ramesh G 2008 Netrin-1 and kidney injury. I. Netrin-1 protects against ischemia-reperfusion injury of the kidney. American Journal of Physiology: Renal Physiology 294 F739-F747. (doi:10.1152/ajpcell.00367.2007)

Woo KY, Santos V \& Gamba M 2013 Understanding diabetic foot ulcers. Nursing 43 36-42; quiz 42-33. (doi:10.1097/01.NURSE. $0000434311.52768 .1 \mathrm{~d})$

Wu YS \& Chen SN 2016 Extracted triterpenes from antrodia cinnamomea reduce the inflammation to promote the wound healing via the STZ inducing hyperglycemia-diabetes mice model. Frontiers in Pharmacology 7 154. (doi:10.3389/fphar.2016.00154)

Xie H, Zou L, Zhu J \& Yang Y 2011 Effects of netrin-1 and netrin-1 knockdown on human umbilical vein endothelial cells and angiogenesis of rat placenta. Placenta 32 546-553. (doi:10.1016/j. placenta.2011.04.003)

Yan J, Tie G, Park B, Yan Y, Nowicki PT \& Messina LM 2009 Recovery from hind limb ischemia is less effective in type 2 than in type 1 diabetic mice: roles of endothelial nitric oxide synthase and endothelial progenitor cells. Journal of Vascular Surgery $\mathbf{5 0}$ 1412-1422. (doi:10.1016/j.jvs.2009.08.007)

Yang X, Li S, Zhong J, Zhang W, Hua X, Li B \& Sun H 2017 CD151 mediates netrin-1-induced angiogenesis through the Src-FAK-Paxillin pathway. Journal of Cellular and Molecular Medicine 21 72-80. (doi:10.1111/jcmm.12939)

Yao J, Xu C, Fang Z, Li Y, Liu H, Wang Y, Xu C \& Sun Y 2016 Androgen receptor regulated microRNA miR-182-5p promotes prostate cancer progression by targeting the ARRDC3/ITGB4 pathway. Biochemical and Biophysical Research Communications 474 213-219. (doi:10.1016/j.bbrc.2016.04.107)

Zhou X, Patel D, Sen S, Shanmugam V, Sidawy A, Mishra L \& Nguyen BN 2017 Poly-ADP-ribose polymerase inhibition enhances ischemic and diabetic wound healing by promoting angiogenesis. Journal of Vascular Surgery 65 1161-1169. (doi:10.1016/j. jvs.2016.03.407)

Zhu KX, Nie SP, Li C, Gong D \& Xie MY 2014 Ganoderma atrum polysaccharide improves aortic relaxation in diabetic rats via PI3K/Akt pathway. Carbohydrate Polymers 103 520-527. (doi:10.1016/j.carbpol.2013.12.080)

Received in final form 17 February 2017 Accepted 1 March 2017
C 2017 Society for Endocrinology Printed in Great Britain 